AN. MED. InTERNA (Madrid) Vol. 18, N. ${ }^{\circ} 10$, pp. 529-530, 2001

\title{
Empiema adquirido en la Comunidad por Acinetobacter baumannii
}

\author{
A. ALONSO FERNÁNDEZ, R. ÁLVAREZ-SALA WALTER, C. PRADOS SÁNCHEZ, \\ S. MAYORALAS ALISES, J. VILLAMOR LEÓN
}

Servicio de Neumología. Hospital La Paz. Universidad Autónoma. Madrid

\author{
ACINETOBACTER BAUMANNII COMMUNITY-ACQUIRED \\ EMPYEMA
}

\begin{abstract}
RESUMEN
Presentamos el caso de un paciente de 44 años de edad, sin ningún antecedente de interés y que ingresa por un cuadro de disnea y fiebre y se le detecta un empiema causado por Acinetobacter baumannii adquirido en la comunidad, lo cual no lo hemos encontrado en la bibliografía.
\end{abstract}

PALABRAS CLAVE: Disnea. Fiebre. Acinetobacter baumannii.

\begin{abstract}
We referred a fourty-four years old male, without interesting perso nal history and who was accepted in the hospital due to dyspnea and fever and who was diagnosed with empyema by Acinetobacter baumannii acquired in the community ; we have found no references about this in the medical literature.
\end{abstract}

KEY WORDS: Dyspnea. Fever. Acinetobacter baumannii.

Alonso Fernández A, Álvarez-Sala Walter R, Prados Sánchez C, Mayoralas Alises S, Villamor León J. Empiema adquirido en la comunidad por Acinetobacter baumannii. An Med Interna (Madrid) 2001; 18: 529-530.

\section{INTRODUCCIÓN}

El Acinetobacter baumannii, conocido anteriormente como Acinetobacter calcoaceticus var anitratus, es un cocobacilo gram negativo de la familia Neiseriaceae que se encuentra ampliamente distribuido por el suelo y en el agua (1), es también un microrganismo comensal de la orofaringe y de la piel de muchas personas sanas. Se ha descrito como el agente causal de muchas neumonías nosocomiales en pacientes inmunocomprometidos y en las unidades de cuidados intensivos. De forma extraordinaria, han sido publicados casos de neumonías extrahospitalarias producidas por Acine tobacter (1-4). En este artículo, presentamos un caso de empiema encapsulado originado por Acinetobacter bauman nii y adquirido en la comunidad, ya que en la literatura revisada no hemos encontrado ninguno.

\section{CASO APORTADO}

Enfermo de 44 años de edad, no fumador, no bebedor ni otros hábitos tóxicos, que ingresa por fiebre y disnea. Entre sus antacedentes personales lo único que destacaba era una neumonía el año anterior. Presentaba desde tres días antes el ingreso fiebre de $39^{\circ} \mathrm{C}$, tos, expectoración purulenta, disnea progresiva hasta hacerse de reposo y dolor en hemitórax derecho de características pleuríticas. En la exploración física se objetivó fiebre de $38,5^{\circ} \mathrm{C}$, taquicardia de $120 \mathrm{lpm}$, hipoventilación en ambas bases pulmonares y crepitantes en campo medio derecho. La gasometría arterial basal detectó $\mathrm{PaO} 2: 50 \mathrm{~mm}$ de $\mathrm{Hg}$; $\mathrm{PaCO}_{2}: 30 \mathrm{~mm}$ de $\mathrm{Hg} ; \mathrm{pH}: 7,47 ; \mathrm{CO} 3 \mathrm{H}: 22 \mathrm{mEq} / \mathrm{l}$. En los análisis de sangre y orina, se apreció una leucocitosis de $26.000 / \mathrm{mm}^{3}$ con una neutrofilia del $91 \%$, el resto de los parámetros fueron normales.

Las baciloscopias y citologías seriadas de esputo y la serología para Legionella, Coxiella, Clamydia y Mycoplasma fueron negativas.

En la radiografía de tórax se objetivó un derrame pleural bilateral en bases, así como una lesión de características extraparenquimatosas en campo medio derecho (Fig. 1a y 1b). La tomografía computarizada tóraco-abdominal detectó adenopatía paratraqueal derecha y otra prevascular de aproximadamente $1,5 \mathrm{~cm}$, así como otra adenopatía menor de un $\mathrm{cm}$ pretraqueal. De igual forma, se confirmó el derrame pleural derecho. Durante los primeros días del ingreso, el paciente tuvo una gran disnea y una importante insuficiencia respiratoria. Se instauró, inicialmente, un tratamiento con Ceftriaxona y Clindamicina sin respuesta clínica, gasométrica ni radiológica. En la toracocentesis se observó cocos y un pH:7,20 en el líquido pleural.

Trabajo aceptado: 31 de Enero de 2000

Correspondencia: A. Alonso Fernández. Marte 32. Tres Cantos 28760. Madrid 


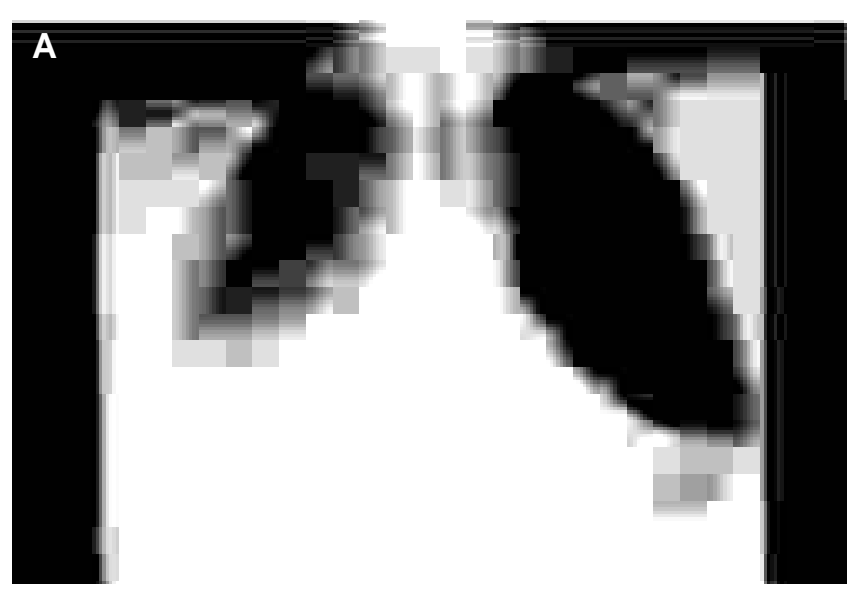

Fig. 1 (A): Radiografía posterio-anterior de tórax que muestra un derrame pleural bilateral y un aumento de densidad en segmento 6 de características extraparenquimatosas.

(B): Radiografía lateral de tórax que confirma las alteraciones des. critas en la figura 1 .

Se llevó a cabo una fibrobroncoscopia en la que no se vieron lesiones endobronquiales. Las citologías y baciloscopias del aspirado bronquial fueron negativos, pero se cultivaron entre 10-100.000 colonias/ml de Acinetobacter baumannii resistente a todos los antibióticos testados, excepto a la Tobramicina y al Imipenem. Después de conocer estos resultados, se retiraron los antibióticos previos y se instauró tratamiento con Imipenem. En una tomografía computarizada, practicada a los 15 días del alta, ya no se observaban las adenopatías previas y sólo persistía un mínimo derrame pleural derecho y un engrosamiento pleural izquierdo. Después de 18 días de tratamineto con Imipenem, el paciente tuvo una buena evolución clínica, radiológica y gasométrica, por lo cual se decidió el alta hospitalaria.

\section{DISCUSIÓN}

Las neumonías nosocomiales causadas por Acinetobacter baumannii están asociadas, la mayor parte de las veces, a intubación endotraqueal, traqueostomía, antibioterapia previa, ingreso en Unidades de Cuidados Intensivos (relacionado con el equipo de ventilación y la colonización del personal) o cirugía reciente. Son excepcionales las neumonías extrahospitalarias desencadenadas por Acinetobacter baumannii.Cuando aparecen suelen

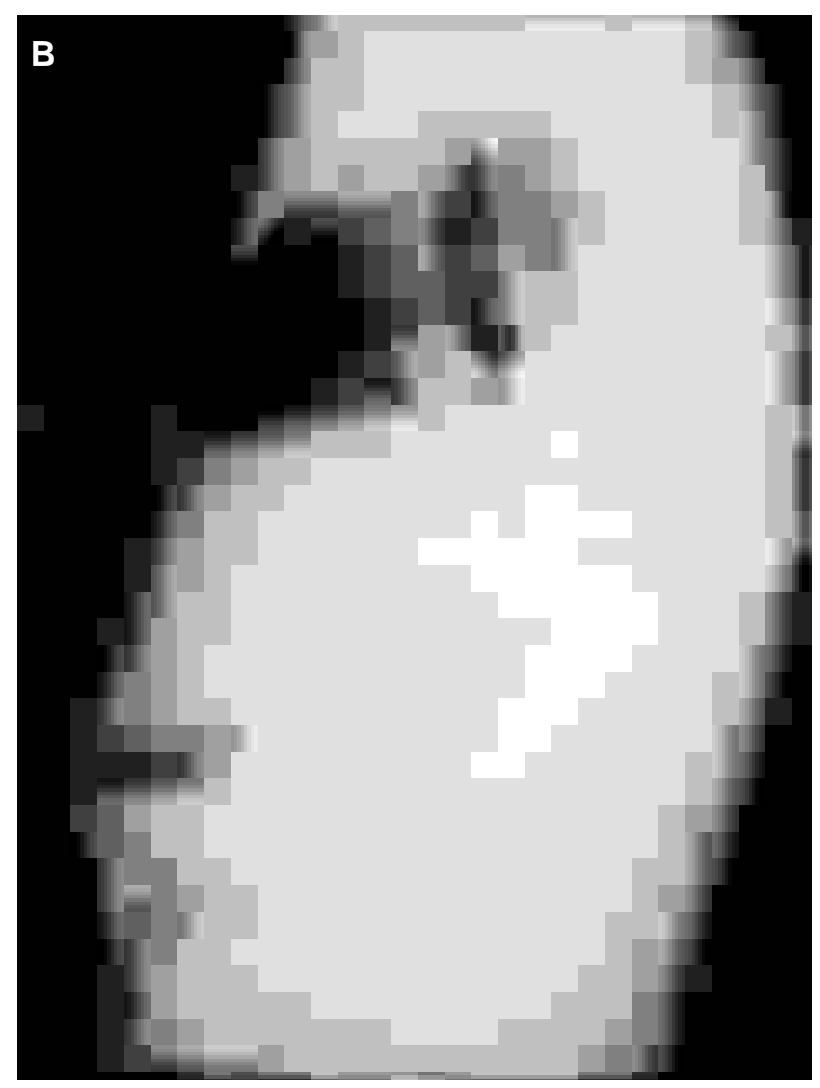

estar en relación con algún factor de riesgo previo como : alcoholismo, tabaquismo, enfermedad pulmonar obstructiva crónica, diabetes mellitus o insuficiencia renal crónica $(2,3)$, ninguno de los cuales los presenta nuestro paciente. Aunque en un estudio de once pacientes de Anstey et al. (2) de 1992, valorando los antecedentes personales de los pacientes,encuentra que seis de ellos $(60 \%)$ habían tenido neumonías previas, aunque no especifica el tiempo transcurrido. Este dato si que está presente en nuestro caso. No obstante, nuestro enfermo tenía además un empiema, lo cual nunca hasta ahora había sido recogido en la literatura revisada. La mortalidad de la neumonía por Acineto bacter baumannii se aproxima al 50\%, (2,3), debido a lo cual es necesario instaurar un tratamiento antibiótico intenso,sensible y precoz. Los antibióticos más apropiados son los aminoglucósidos, sobre todo la gentamicina, penicilinas con actividad antipseudomona y el imipenem (2-4); sin embargo, el tratamiento debe ajustarse, en lo posible, a los resultados del antibiograma y con una duración de al menos tres semanas.

Pensamos que en una neumonía con empiema que no responde a los tratamientos antibióticos convencionales hay que tener en cuenta, por supuesto entre otros gérmenes, al Acine tobacter baumanni.

\section{Bibliografía}

1. Goodhart G, Abratyn E, Watson R, Root R, Egert J. Community acquired Acinetobacter calcoaceticus var anitratus pneumonia. JAMA 1977;238:1516-8.

2. Anstey N, Currie B, Withnall K. Community acquired Acinetobacter pneumonia in the Nothern territory of Australia.Clin Infec Dis
1992;14:83-91.

3. Bilgiç H, Akin S, Tasan Y, Ekiz K, Seber O. A case of Acinetobacter calcoaceticus pneumonia. Thorax 1995;50:315-6.

4. Bick J, Semel J. Fulminant Community-acquired Acinetobacter pneumonia in a Healthy Woman. Clin Infec Dis 1993; 17: 820-1. 